$\square$ Math-Net.Ru

Общероссийский математический портал 
И. Ю. Доманов, О спектральной кратности некоторых вольтерровых операторов в соболевских пространствах, Матем. заметки, 2002, том 72, выпуск 2, 306-311

DOI: https://doi.org/10.4213/mzm662

Использование Общероссийского математического портала Math-Net.Ru подразумевает, что вы прочитали и согласны с пользовательским соглашением http://www. mathnet.ru/rus/agreement 
Параметры загрузки:

IP: 3.82 .47 .9

26 апреля 2023 г., 17:11:49

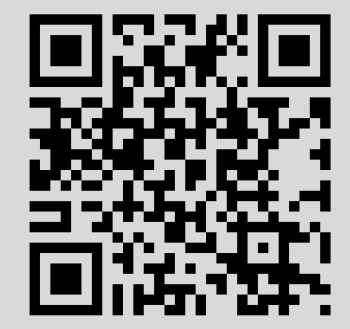




\section{О СПЕКТРАЛЬНОЙ КРАТНОСТИ НЕКОТОРЫХ ВОЛЬТЕРРОВЫХ ОПЕРАТОРОВ В СОБОЛЕВСКИХ ПРОСТРАНСТВАХ}

\section{И. Ю. Доманов}

Введение. Прежде всего дадим несколько определений.

ОПРЕДЕЛЕниЕ 1. Подпространство $E$ банахова пространства $X$ назьвают ииклическим подпространством для оператора $T \in[X]$, если $\operatorname{span}\left\{T^{n} E: n \geqslant 0\right\}=X$. Вектор $f(\in X)$ называют ииклическим вектором для оператора $T$, если $\operatorname{span}\left\{T^{n} f: n \geqslant 0\right\}=X$. Множество всех циклических подпространств оператора $T$ обозначают Сус $(T)$.

ОПРЕДЕЛЕНИЕ 2. Положим

$$
\mu_{T}:=\inf _{E}\{\operatorname{dim} E: E-\text { циклическое подпространство оператора } T \text { в } X\},
$$

число $\mu_{T}$ называют спектральной кратностью оператора $T$ в $X$.

Отметим, что $\mu_{T}$ может быть равно $\infty$. Ясно, что оператор $T$ цик лический тогда и только тогда, когда $\mu_{T}=1$. 
Отметим, что понятие спектральной кратности играет важную роль в теории управления [1]. Именно, линейная динамическая система описывается уравнением

$$
\frac{d x(t)}{d t}=A x(t)+B u(t), \quad t \geqslant 0
$$

с операторами $A \in[X], B=B \uparrow U, B \in[U, X]$, где $X$ и $U$-банаховы пространства (пространство состояний и управляющее подпространство соответственно) и $x(t) \in X, u(t) \in U$.

Первая из задач теории управления заключается в отыскании всех пространств $U$, удовлетворяюших следующему свойству управляемости: для любого состояния $x \in X$, любого $\varepsilon>0$ и любого момента времени $t>0$ найдется (гладкая) функция управления $u(\cdot)$, при которой решение системы (1) достигает $\varepsilon$-окрестности $x,\|x-x(t)\|<\varepsilon$, начиная из некоторого фиксированного исходного состояния $x(0)$ (например, $x(0)=0$ ).

Хорошо известная теорема Калмана утверждает, что система $A, B \uparrow U$ управляема тогда и только тогда, когда, $B U \in$ Сус $A$, т.е. когда минимальная размерность управляющего подпространства в (1) равна $\mu_{A}$.

Исследуя другие задачи теории управления, Никольский и Васюнин [2] ввели еще одну характеристику оператора $A$.

ОПРЕДЕЛЕНИЕ 3 [2]. Положим

$$
\operatorname{disc} T:=\sup _{E \in \operatorname{Cyc} T} \min \left\{\operatorname{dim} E^{\prime}: E^{\prime} \subset E, E^{\prime} \in \operatorname{Cyc} T\right\}
$$

$\operatorname{disc} T$ назьвают disc-характеристикой оператора $T$ ( "disc" - аббревиатура от "Dimension of the Input Subspace of Control").

Ясно, что $\operatorname{disc} A \geqslant \mu_{A}$. Отметим, что как $\operatorname{disc} A$, так и $\mu_{A}$ зависят только от решетки инвариантных подпространств Lat $A$ оператора $A$.

Как обычно, $W_{p}^{k}[0,1]$ обозначает соболевское пространство: $f \in W_{p}^{k}[0,1]$, если $f$ имеет $(k-1)$-ю абсолютно-непрерьвную производную и $f^{(k)} \in L_{p}[0,1] ; W_{p, 0}^{k}[0,1]=\left\{f \in W_{p}^{k}[0,1]: f(0)=\cdots\right.$ $\left.=f^{(k-1)}(0)=0\right\}$. Положим $W_{p}^{0}[0,1]=W_{p, 0}^{0}[0,1]:=L_{p}[0,1]$.

Хорошо известно [3], [4], что оператор

$$
J^{\alpha}: f(x) \rightarrow \int_{0}^{x} f(t) \frac{(x-t)^{\alpha-1}}{\Gamma(\alpha)} d t, \quad \operatorname{Re} \alpha>0,
$$

является одноклеточным в пространстве $L_{p}[0,1], 1 \leqslant p<\infty$, и его решетка инвариантных подпространств Lat $J^{\alpha}$ имеет вид

$$
\text { Lat } J^{\alpha}=\left\{E_{a}: \chi_{[0, a]} L_{p}[0,1]: 0 \leqslant a \leqslant 1\right\} .
$$

Из (2) следует, что вектор $f \in \mathrm{Cус} J^{\alpha}$ тогда и только тогда, когда

$$
\int_{0}^{\varepsilon}|f(x)|^{p} d x>0 \quad \forall \varepsilon>0 .
$$

Это условие назьвают $\varepsilon$-условием.

Цекановским было получено описание решетки инвариантных подпространств Lat $J_{k}$ оператора интегрирования $J_{k}$, действующего в пространстве Соболева $W_{2}^{k}[0,1]$. Именно, в [5] показано, что оператор интегрирования $J_{k}: f \rightarrow \int_{0}^{x} f(t) d t$ в $W_{2}^{k}[0,1]$ также одноклеточен и решетка Lat $J_{k}$ состоит из непрерьвной $\mathrm{Lat}^{c} J_{k}$ и дискретной Lat ${ }^{d} J_{k}$ частей: Lat $J_{k}=\mathrm{Lat}^{c} J_{k} \cup \mathrm{Lat}^{d} J_{k}$. Здесь

$$
\text { Lat }^{c} J_{k}=\left\{E_{a}:=\left\{f: f \in W_{p}^{k}[0,1], f=0 \text { при } x \in[0, a]\right\}, 0 \leqslant a \leqslant 1\right\}
$$

- непрерьвная часть и $\mathrm{Lat}^{d} J_{k}=\left\{E_{l}^{k}\right\}_{l=0}^{k}$ с $E_{k}^{k}:=W_{p}^{k}[0,1]$ и с

$$
E_{l}^{k}=\left\{f \in W_{p}^{k}[0,1]: f(0)=\cdots=f^{(k-l-1)}(0)=0\right\}, \quad l \in\{0,1, \ldots, k-1\},
$$


- дискретная часть. Ясно, что $W_{p, 0}^{k}[0,1]=E_{0}^{k} \subset E_{1}^{k} \subset \cdots \subset E_{k}^{k}=W_{p}^{k}[0,1]$.

В работе [6] показано, что спектральная кратность $\mu_{J_{k}^{\alpha}}$ оператора $J_{k}^{\alpha}$ равна $\mu_{J_{k}^{\alpha}}=\min (-[\alpha], k)$ и, следовательно, $J_{k}^{\alpha}$ цикличен тогда, когда либо $\alpha=1$, либо $k=1$. Там же, в [6], получено описание множества Сус $J_{k}^{\alpha}$.

Описание циклических и инвариантных подпространств оператора $A:=J^{\alpha} \otimes B$, действующего в пространствах вектор-функций $L_{p}[0,1] \otimes \mathbb{C}^{n}$, где $B=\operatorname{diag}\left(\lambda_{1}, \ldots, \lambda_{n}\right)$ - диагональная матрица, были получены Маламудом [7]. В частности, в [7] показано, что оператор $A$ цик личен только тогда, когда

$$
\arg \lambda_{i} \neq \arg \lambda_{j} \quad \text { при } i \neq j .
$$

Там же, в [7], показано, что при условии (5) решетка Lat $A$ распадается:

$$
\text { Lat } A=\bigoplus_{j=1}^{n} \text { Lat } \lambda_{j} J^{\alpha}=\left\{\bigoplus_{i=1}^{n} E_{a_{i}}^{p}: 0 \leqslant a_{i} \leqslant 1\right\} \text {. }
$$

В [8] эти результаты были частично обобщены на случай соболевских пространств вектор функций. Именно в [8] получено описание цик лических подпространств для оператора $J \otimes B$, действующего в пространствах $\bigoplus_{j=1}^{n} W_{p}^{k}[0,1]$ и $\bigoplus_{j=1}^{n} W_{p, 0}^{k}[0,1]$. В настоящем сообщении некоторые результаты из [7] и [8] распространяются на случай оператора $J^{\alpha} \otimes B$, действующего в пространстве $\bigoplus_{i=1}^{l} W_{p}^{k}[0,1]$.

Мы начнем с более простого описания Сус $A_{k, 0}$ для оператора $A_{k, 0}$, являющегося сужением оператора $A_{k}$ на подпространство $\bigoplus_{j=1}^{n} W_{p, 0}^{k}[0,1]$.

Циклические подпространства оператора $A_{k, 0}$. Обозначим через $J_{k, 0}^{\alpha}$ и $J_{k}^{\alpha}:=J_{k, k}^{\alpha}$ оператор $J^{\alpha}$, действующий в пространствах $W_{p, 0}^{k}[0,1]$ и $W_{p}^{k}[0,1]$ соответственно. Пусть также $J_{k, l}^{\alpha}$ обозначает сужения оператора $J_{k}^{\alpha}$ на подпространства $E_{l}^{k}$, определенные соотношениями (4) $(l \leqslant k-1)$ и $E_{k}^{k}:=W_{p}^{k}[0,1]$.

Оператор $J_{k}^{\alpha}$ определен корректно при $\alpha \in \mathbb{Z}_{+} \backslash\{0\}$ или $\operatorname{Re} \alpha>k-1 / p$, а его сужение $J_{k, 0}^{\alpha}$ на подпространство $W_{k, 0}^{k}[0,1]$ определено при $\operatorname{Re} \alpha>0$.

Tеорема 4. Пусть $B=\operatorname{diag}\left(\lambda_{1}, \ldots, \lambda_{n}\right)-$ диагональная матрица с собственными значениями, имеюшими несовпадающие аргументы $\left(\arg \lambda_{i} \neq \arg \lambda_{j} n p u i \neq j\right), u A_{k, 0}=$ $\bigoplus_{i=1}^{n} \lambda_{i} J_{k, 0}^{\alpha}$. Тогда система $\left\{f_{i}\right\}_{i=1}^{N}$ векторов $f_{i}=\bigoplus_{j=1}^{n} f_{i j}$ порохсдает ииклическое подпространство в $W_{p, 0}^{k}[0,1] \otimes \mathbb{C}^{n}$ для оператора $A_{k, 0}$ тогда и только тогда, когда каждая из функций

$$
F_{j}:=\sum_{i=1}^{N}\left|f_{i j}(x)\right|, \quad i \in\{1, \ldots, n\}
$$

удовлетворяет в-условию (3).

$B$ частности, оператор $A_{k, 0}$ ииклический в $W_{p, 0}^{k}[0,1] \otimes \mathbb{C}^{n}$ u $f=\left(f_{1}, \ldots, f_{n}\right) \in \mathrm{Cyc} A_{k, 0}$ тогда и только тогда, когда $f_{i}(x)$ удовлетворяет $\varepsilon$-условию (3) при всех $i \in\{1, \ldots, n\}$.

Для формулировки следующей теоремы нам понадобится

ОпРЕДЕЛЕНИЕ 5 [7]. Определитель функциональной матрицы

$$
F(x)=\left(f_{i j}(x)\right)_{i, j=1}^{n}, \quad f_{i j} \in W_{p}^{k}[0,1],
$$

подсчитанный по отношению к свертке

$$
(f * g)(x)=\int_{0}^{x} f(x-t) g(t) d t=\int_{0}^{x} g(x-t) f(t) d t=(g * f)(x),
$$

назьвают *-определителем и обозначают $*$-det $F(x)$.

Аналогично *-миноры $F(x)$ - это миноры, подсчитанные по отношению к свертке; $*$-rank $F(x)$ это максимальный ранг *-миноров матрицы $F(x)$, удовлетворяющих $\varepsilon$-условию (3). 
Теорема 6. Пусть $\alpha>0$ и В - диагональная матрича вида

$$
\begin{gathered}
B=\bigoplus_{j=1}^{r} \lambda_{j} I_{n_{j}}, \quad \arg \lambda_{j}=\arg \lambda_{1} \quad \forall j \in\{1, \ldots, r\}, \quad n=n_{1}+\cdots+n_{r}, \\
\lambda_{j}=\lambda_{1} / s_{j}^{\alpha}, 1=s_{1} \leqslant s_{2} \leqslant \ldots \leqslant s_{r}, u \\
f_{i j}=\left\{f_{i j 1}, \ldots, f_{i j n_{j}}\right\} \in W_{p, 0}^{k}[0,1] \otimes \mathbb{C}^{n_{j}}, \quad 1 \leqslant i \leqslant N, \quad 1 \leqslant j \leqslant r .
\end{gathered}
$$

Тогда $\mu_{A_{k, 0}}=n$ и система $\left\{f_{i}\right\}_{i=1}^{N}$ векторов $f_{i}=\bigoplus_{j=1}^{r} f_{i j}$ порожсдает ииклическое подпространство $\bigoplus_{j=1}^{n} W_{p, 0}^{k_{j}}[0,1]$ для оператора

$$
A_{k, 0}=J_{k, 0}^{\alpha} \otimes B=\bigoplus_{j=1}^{r} \lambda_{j} J_{k, 0}^{\alpha} \otimes I_{n_{j}}
$$

тогда и только тогда, когда выполнены следующие условия:

1) $N \geqslant n$

2) матрича

$$
F_{r}(x)=\left(\begin{array}{cccc}
f_{11}\left(s_{1} x\right) & f_{12}\left(s_{2} x\right) & \ldots & f_{1 r}\left(s_{r} x\right) \\
\ldots & \ldots & \ldots & \ldots \\
f_{r 1}\left(s_{1} x\right) & f_{r 2}\left(s_{2} x\right) & \ldots & f_{r r}\left(s_{r} x\right)
\end{array}\right)
$$

имеет максимальный *-ранг, а именно

$$
*-\operatorname{rank} F_{r}(x)=n_{1}+n_{2}+\cdots+n_{r}
$$

Прежде чем дать описание множества Сус $A_{k, 0}$ в случае произвольного оператора

$$
A_{k, 0}:=J_{k}^{\alpha} \otimes B=\lambda_{1} J_{k_{1}, 0}^{\alpha} \oplus \cdots \oplus \lambda_{n} J_{k_{n}, 0}^{\alpha},
$$

введем следующее обозначение. Пусть $T_{i} \in\left[H_{i}\right], i=\overline{1, n}, T=\bigoplus_{i=1}^{n} T_{i}, H=\bigoplus_{i=1}^{n} H_{i}$. Легко видеть, что если $E \in \mathrm{Cус} T$, то $P_{i} E \in$ Сус $T_{i}$, где $P_{i}$ - естественная проекция $H$ на $H_{i}$. Если верно обратное, т.е. если соотношения $P_{i} E \in$ Сус $T_{i}, i=\overline{1, n}$, влекут $E \in$ Сус $T$, то будем писать Сус $T=\bigvee_{i=1}^{n} \operatorname{Cyc~} T_{i}$. Это условие, очевидно, вьполняется, если Lat $T=\bigoplus_{i=1}^{n} \operatorname{Lat} T_{i}$.

Tеорема 7. Пусть $\alpha>0$, оператор $A_{j, 0}:=\bigoplus_{i=1}^{n_{j}} \lambda_{i j} J_{k_{i j}, 0}^{\alpha}$ определен в пространстве $\bigoplus_{i=1}^{n_{j}} W_{p, 0}^{k_{i j}}[0,1], 1 \leqslant j \leqslant r, u A_{0}:=\bigoplus_{j=1}^{r} A_{j, 0}$. Пусть maжже $\lambda_{i j}=\lambda_{1 j} s_{i j}^{\alpha}, 1=$ $s_{1 j} \leqslant s_{2 j} \leqslant \cdots \leqslant s_{n_{j} j} u \arg \lambda_{1 j} \neq \arg \lambda_{1 l}(\bmod 2 \pi)$ npu $j \neq l$. Tогдa

1) $\operatorname{Cyc} A_{0}=\bigvee_{j=1}^{r} \operatorname{Cyc} A_{j, 0}$;

2) $\mu_{A_{0}}=\max \left\{n_{j}: 1 \leqslant j \leqslant r\right\}$

3) система $\left\{f_{i}\right\}_{i=1}^{N}$ векторов $f_{i}=\left\{f_{i 11}, \ldots, f_{i 1 n_{1}}, \ldots, f_{i r 1}, \ldots, f_{i r n_{r}}\right\}$ порождает чиклическое подпространство тогда и только тогда, когда матрици

$$
F_{j}(x)=\left(\begin{array}{ccc}
f_{1 j 1}\left(s_{1 j} x\right) & \ldots & f_{1 j n_{j}}\left(s_{n_{j} j} x\right) \\
\ldots & \ldots & \ldots \\
f_{N j 1}\left(s_{1 j} x\right) & \ldots & f_{N j n_{j}}\left(s_{n_{j} j} x\right)
\end{array}\right)
$$

имеют максимальный $*$-ране, а именно $*-\operatorname{rank} F_{j}(x)=n_{j}$.

ЗАмЕчаниЕ 8 . Теоремы 4, 6,7 обобщают соответствующие результаты из [7] и совпадают с ними при $k=0$, т.е. для пространства $W_{p, 0}^{0}:=L_{p}[0,1]$.

Циклические подпространства оператора $A_{k}$. Для каждой системы векторов $\varphi=$ $\left\{\vec{\varphi}_{i}\right\}_{1}^{m}$ обозначим через $W(\varphi)(n \times m)$-матрицу, состоящую из столбцов $\vec{\varphi}_{i}: W(\varphi)=\left(\vec{\varphi}_{1}, \ldots, \vec{\varphi}_{m}\right)$. 
Лемма 9. Пусть $m_{i}=\min \left(-[-\alpha], k_{i}\right), k_{i} \in \mathbb{Z}_{+} \backslash\{0\}, u \lambda_{i} \neq 0$ для $i=\{1, \ldots, n\}$. Тогдa система векторов

$$
\vec{\varphi}_{i}=\operatorname{col}\left(\varphi_{i 11}, \ldots, \varphi_{i 1 k_{1}}, \varphi_{i 21}, \ldots, \varphi_{i 2 k_{2}}, \ldots \ldots \varphi_{i n 1}, \ldots, \varphi_{i n k_{n}}\right), \quad i=\overline{1, N}
$$

порождает ииклическое подпространство для оператора $A=\lambda_{1} J\left(0 ; k_{1}\right)^{\alpha} \oplus \cdots \oplus$ $\lambda_{n} J\left(0, k_{n}\right)^{\alpha}$ в пространстве $\mathbb{C}^{k}, k=k_{1}+\cdots+k_{n}$, тогда и только тогда, когда

1) $N \geqslant \sum_{i=1}^{n} m_{i}$

2) матрица $W_{0}=P W(\varphi)$ имеет максимальный ране, m.е. $\operatorname{rank} W_{0}=\sum_{i=1}^{n} m_{i}$.

TEOPEMA 10. Пусть $m=\min (-[-\alpha], k), k \geqslant 1$. Тогдa

1) система векторов $f_{i}(x)=\left\{f_{i 1}(x), \ldots, f_{i n}(x)\right\}, i=\overline{1, N}$, порождает ииклическое подпространство для оператора $A_{k}=\lambda_{1} J_{k}^{\alpha} \oplus \cdots \oplus \lambda_{n} J_{k}^{\alpha}$ в пространстве $\bigoplus_{i=1}^{n} W_{p}^{k}[0,1]$ тогда и только тогда, когда выполнень следующие условия:

i) $N \geqslant m n$;

ii) матрича

$$
W(0)=\left(\begin{array}{cccc}
f_{11}(0) & f_{21}(0) & \ldots & f_{N 1}(0) \\
f_{11}^{\prime}(0) & f_{21}^{\prime}(0) & \ldots & f_{N 1}^{\prime}(0) \\
f_{11}^{(m-\dot{1})}(0) & f_{21}^{(m-\dot{1})}(0) & \ldots & f_{N 1}^{(m-1} \cdots(0) \\
\ldots & \ldots & \ldots & \ldots \\
f_{1 n}(0) & f_{2 n}(0) & \ldots & f_{N n}(0) \\
f_{1 n}^{\prime}(0) & f_{2 n}^{\prime}(0) & \ldots & f_{N n}^{\prime}(0) \\
\left.f_{1 n}^{(m-1} \dot{1}\right)(0) & f_{2 n}^{(m-1)}(0) & \ldots & \left.f_{N n}^{(m-1} \dot{1}\right)(0)
\end{array}\right)
$$

имеет максимальныц ианг, m.е. $\operatorname{rank} W(0)=m n$;

2) $\mu_{A_{k}}=m n$

3) $\operatorname{disc} A_{k}=m n$.

ЗАмеч ание 11. Отметим, что описания Сус $A_{k, 0}$ и Сус $A_{k}$ в пространствах $\bigoplus_{j=1}^{n} W_{p, 0}^{k}[0,1]$ и $\bigoplus_{j=1}^{n} W_{p}^{k}[0,1]$ существенно различны.

ЗАмечание 12 . Для $\alpha=1$, т.е. для оператора $A_{k}=\lambda_{1} J_{k} \oplus \cdots \oplus \lambda_{n} J_{k}$, теорема 10 получена в [8]. Для оператора $J^{\alpha}$, действующего в пространстве $W_{p}^{k}[0,1]$ (т.е. при $n=1$ ), теорема 10 доказана в [6].

Объединяя теоремы 7,10 , приходим к основному результату заметки.

TEOPEмA 13. Пусть либо $\alpha \in \mathbb{Z}_{+} \backslash\{0\}$, либо $\alpha>\max _{j}\left(k_{j}-1 / p\right)$. Положим

$$
W_{1}=\bigoplus_{i=1}^{l} W_{p}^{k_{i}}[0,1], \quad W_{1,0}=\bigoplus_{i=1}^{l} W_{p, 0}^{k_{i}}[0,1], \quad W_{2,0}=\bigoplus_{i=l+1}^{n} W_{p, 0}^{k_{i}}[0,1]
$$

Пусть также операторь

$$
A_{1}=\bigoplus_{i=1}^{l} \lambda_{i} J_{k_{i}}^{\alpha}, \quad A_{1,0}=\bigoplus_{i=1}^{l} \lambda_{i} J_{k_{i}, 0}^{\alpha}, \quad A_{2,0}=\bigoplus_{i=l+1}^{n} \lambda_{i} J_{k_{i}, 0}^{\alpha}
$$

действуют в пространствах $W_{1}, W_{1,0}, W_{2,0}$ соответственно. Тогда

$$
\operatorname{Cyc}\left(A_{1} \oplus A_{2,0}\right)=\operatorname{Cyc}\left(A_{1,0} \oplus A_{2,0}\right) \cap\left(\operatorname{Cyc} A_{1} \oplus W_{2,0}\right),
$$


где мнохества $\mathrm{Cyc}\left(A_{1,0} \oplus A_{2,0}\right)$ и Сус $A_{1}$ описаны в теоремах $6,7,10$, соответственно. В частности, если $\alpha<k_{i}-1$ (в этом случае $\alpha \in \mathbb{Z}_{+}$) для $i=\overline{1, l}$, то система векторов $f_{i}=\left\{f_{i 1}, \ldots, f_{i n}\right\}, i=1,2 \ldots, N$, порождает ииклическое подпространство для оператора $A$, если выполнены следующие условия:

1) матрица

$$
W(0)=\left(\begin{array}{cccc}
f_{11}(0) & f_{21}(0) & \ldots & f_{N 1}(0) \\
f_{11}^{\prime}(0) & f_{21}^{\prime}(0) & \ldots & f_{N 1}^{\prime}(0) \\
f_{11}^{(\alpha-i)}(0) & f_{21}^{(\alpha-1}(0) & \ldots & f_{N 1}^{(\alpha-1)}(0) \\
\ldots & \ldots & \ldots & \ldots \\
f_{1 l}^{\prime}(0) & f_{2 l}^{\prime}(0) & \ldots & f_{N l}(0) \\
f_{1 l}^{\prime}(0) & f_{2 l}^{\prime}(0) & \ldots & f_{N l}^{\prime}(0) \\
f_{1 l}^{(\alpha-i)}(0) & \left.f_{2 l}^{(\alpha-1} \dot{1}\right)(0) & \ldots & f_{N l}^{(\alpha-1)}(0)
\end{array}\right)
$$

имеет максимальныц ранг, т.е. $\operatorname{rank} W(0)=\alpha l$;

2) имеет место оченка

$$
N \geqslant\left\{\begin{array}{lll}
\alpha l, & \text { ecлu } \arg \lambda_{i} \neq \arg \lambda_{j}, & 1 \leqslant i, j \leqslant n, \\
\max (\alpha l, n), & \text { ecлu } \arg \lambda_{i} \leqslant \arg \lambda_{j}, & 1 \leqslant i \leqslant j \leqslant n ;
\end{array}\right.
$$

3а) в случае $\arg \lambda_{i} \neq \arg \lambda_{j}, 1 \leqslant i, j \leqslant n$, каждая из функиий $F_{j}=\sum_{i=1}^{N}\left|f_{i j}(x)\right|$ удовлетворяет в-условию (3);

3b) в случае $\arg \lambda_{i} \leqslant \arg \lambda_{j}, 1 \leqslant i \leqslant j \leqslant n$, матрица

$$
F(x)=\left(\begin{array}{cccc}
f_{11}\left(\lambda_{1} x / \lambda_{2}\right) & f_{12}\left(\lambda_{1} x / \lambda_{3}\right) & \ldots & f_{1 n}\left(\lambda_{1} x / \lambda_{n}\right) \\
\ldots & \ldots & \ldots & \ldots \\
f_{N 1}\left(\lambda_{1} x / \lambda_{2}\right) & f_{N 2}\left(\lambda_{1} x / \lambda_{3}\right) & \ldots & f_{N n}\left(\lambda_{1} x / \lambda_{n}\right)
\end{array}\right)
$$

имеет максимальный *-ранг, а именно $*-\operatorname{rank} F(x)=n$.

\section{СПИСОК ЦИТИРОВАННОЙ ЛИТЕРАТУРЫ}

1. Wonham M.W. Linear Multivariable Control. Berlin: Springer-Verlag, 1974. 2. Nikolskii N. K., Vasjunin V. I. // J. Operator Theory. 1981. V. 10. Р. 307-330. 3. Гохберг И. Ц., Крейн М.Г. Теория вольтерровых операторов в гильбертовом пространстве и ее приложения. М.: Наука, 1967. 4. Никольский Н. К. Лекции об операторе сдвига. М.: Наука, 1980. 5. Цекановский Э. Р. // УМН. 1965. Т. 20. №6. С. 169-172. 6. Domanov I. Yu., Malamud M. M. Invariant and hyperinvariant subspaces of an operator $J^{\alpha}$ and related operator algebras in Sobolev spaces // Linear Algebra and Appl. (to appear). 7. Malamud M. M. // Operator Theory Advances Appl. Integral and Differential Operators. 1998. V. 102. P. 143-167. 8. Domanov I. Yu. // Methods of Functional Analysis and Topology. 1999. V. 1. № 5. P. 1-12. 